\title{
Measurement of Residual Stresses in Different Thicknesses of Laser Shock Peened Aluminium Alloy Samples
}

\author{
S.N van Staden ${ }^{1,2, a^{*}}$, C. Polese ${ }^{1,2, b}$, D. Glaser ${ }^{3, c}$, J.-P. Nobre $^{1,2, d}$, A.M. Venter ${ }^{2,4, e}$, \\ D. Marais ${ }^{4, f}$, J. Okasinski ${ }^{5, g}$ and J.-S. Park ${ }^{5, h}$
}

${ }^{1}$ School of Mechanical, Industrial and Aeronautical Engineering, University of the Witwatersrand, 1 Jan Smuts Avenue, Johannesburg, 2000, South Africa

${ }^{2}$ DST-NRF Centre of Excellence in Strong Materials, University of the Witwatersrand, 1 Jan Smuts Avenue, Johannesburg, 2000, South Africa

${ }^{3}$ CSIR National Laser Centre, Meiring Naudé Road, Pretoria, 0184, South Africa

${ }^{4}$ Research and Development Division, Necsa SOC Limited, R104 Pelindaba, Pretoria, 0240, South Africa

${ }^{5}$ Advanced Photon Source, Argonne National Laboratory, 9700 S. Cass Avenue, Lemont, Illinois, 60439, USA

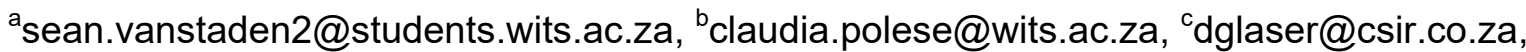
djoaopaulo.nobre@wits.ac.za, ${ }^{\mathrm{e}}$ andrew.venter@necsa.co.za, ${ }^{f}$ deon.marais@necsa.co.za,

gokasinski@aps.anl.gov, hparkjs@aps.anl.gov

Keywords: Laser Shock Peening, Aluminium, Residual Stress, Incremental Hole Drilling, X-ray Diffraction, Synchrotron, Energy-Dispersive, Neutron Diffraction

\begin{abstract}
This study focused on depth-resolved residual stress results determined with a number of complementary techniques on Laser Shock Peening (LSP) treated aluminium alloy 7075-T651 samples with different thicknesses $(6 \mathrm{~mm}$ and $1.6 \mathrm{~mm})$. Samples were prepared from a single commercially produced rolled plate that was then treated with LSP. Residual stresses were measured using Laboratory X-Ray Diffraction (LXRD), Incremental Hole Drilling (IHD), Neutron Diffraction (ND) and Synchrotron XRD (SXRD). The LSP treatment resulted in the establishment of compressive residual stresses that varied rapidly in the near surface region. The compressive stresses extended up to $1.5 \mathrm{~mm}$ in depth in the $6 \mathrm{~mm}$ thick sample. Some surface stress relaxation was observed in the first $25 \mu \mathrm{m}$, but substantially large stresses existed at 50 $\mu \mathrm{m}$. This investigation strongly motivated why residual stress profiles should be obtained using a variety of techniques.
\end{abstract}

\section{Introduction}

Laser Shock Peening (LSP) is a surface treatment technique that involves the ablation of a metal sample by pulsed, high intensity, laser irradiation. The sample surface is covered by some medium transparent to the laser (typically water). A plasma is formed at the surface due to the rapid heating by the laser. This event is confined by the transparent medium so, as the plasma expands, it generates extremely high pressures which are transferred to the sample through shock waves. These plastically deform the sample and are expected to establish a beneficial compressive residual stress near the surface [1].

Such a compressive residual stress field can result in slowed fatigue crack propagation and increased fatigue life [2], improved resistance to stress corrosion cracking [3], as well as permanent deformation of the samples [4]. LSP has been shown to be more effective than more 
traditional shot peening because the magnitude and depth of compression induced by LSP are greater [5]. LSP therefore has many potential applications, such as in the aerospace industry.

There are a number of LSP parameters that can be varied so as to optimize the process for different applications, e.g. power intensity, laser spot size and coverage. As part of the development of a particular laser system, it is important to understand the effect these parameters have on the induced residual stress field for various alloys and sample thicknesses. Of particular interest are the surface residual stress, the maximum compressive residual stress, as well as the depth to which the residual stresses are compressive.

In order to obtain a depth-resolved residual stress profile that can reveal this information, a number of complementary residual stress measurement techniques need to be used [6]. These include: Laboratory X-Ray Diffraction (LXRD) which can non-destructively measure near the surface (about $25 \mu \mathrm{m}$ ); Neutron Diffraction (ND) and Synchrotron XRD (SXRD) which can nondestructively measure through the entire depth of most materials (but with a larger gauge volume so the results are averaged over a greater depth); Incremental Hole Drilling (IHD) which can measure semi-destructively to an intermediate depth $(1-2 \mathrm{~mm})$, but is only accurate in samples thicker than $5.13 \mathrm{~mm}$ for conventional IHD strain gauge rosettes.

The objective of this study was therefore to compare the residual stress results in two LSP treated aluminium alloy 7075 (an aeronautical alloy) plates with different thicknesses investigated with a number of complementary techniques.

\section{Methodology}

Sample Preparation. Aluminium alloy 7075-T651 plate samples with dimensions $60 \mathrm{~mm}$ x 60 $\mathrm{mm}$ were prepared from a single $15 \mathrm{~mm}$ thick rolled plate. One side of the samples was machined to remove $1 \mathrm{~mm}$ followed by machining on the opposite side to respective thicknesses of $6 \mathrm{~mm}$ and $1.6 \mathrm{~mm}$, as shown in Fig. 1a. This approach was followed so that the LSP treatment was performed on a similarly prepared surface (the one with $1 \mathrm{~mm}$ removed) for consistency. The bulk elastic constants were taken as $\mathrm{E}=71.7 \mathrm{GPa}$ and $v=0.33$ and the corresponding diffraction elastic constants for the $\{311\}$ lattice plane as: $\mathrm{S}_{1}=-5.158 \times 10^{-6} \mathrm{MPa}^{-1}$ and $1 / 2 \mathrm{~S}_{2}=$ $1.957 \times 10^{-5} \mathrm{MPa}^{-1}$.

a)

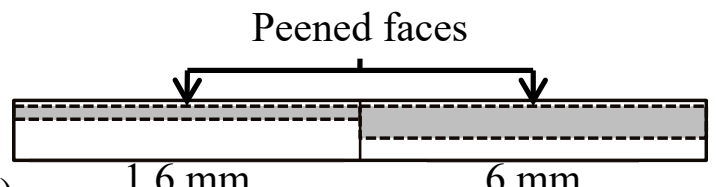

Fig. 1: a)Sample Preparation, b) Sample Geometry and LSP patches, c) raster pattern indicating the laser step and scan directions.

Laser Shock Peening. LSP was performed at the National Laser Centre of the CSIR, South Africa, using an Nd:YAG laser system with a wavelength of $1064 \mathrm{~nm}$ and a pulse frequency of $20 \mathrm{~Hz}$. The confinement medium was flowing water and no protective coating was used. The LSP parameters were as follows: a power intensity of $3 \mathrm{GW} / \mathrm{cm}^{2}$, a spot diameter of $1.5 \mathrm{~mm}$ and a coverage of 500 spots $/ \mathrm{cm}^{2}$. Four $13 \mathrm{~mm}$ x $14 \mathrm{~mm}$ LSP patches were applied to the sample, as shown in Fig 1b, and the LSP was performed in a raster pattern, as shown in Fig. 1c. The X-and $y$-directions used in subsequent sections are aligned with the step and scan directions respectively and the z-direction is normal to these, through the depth of the samples.

Laboratory X-Ray Diffraction. LXRD measurements were performed on the $6 \mathrm{~mm}$ thick sample (with and without LSP) using a Bruker D8 Discover at Necsa, South Africa. X-rays from 
a $\mathrm{Cu}-\mathrm{K} \alpha$ source with a wavelength of $1.54 \AA$ and a beam size of $0.8 \mathrm{~mm}$ were used to perform the measurements in the $\sin ^{2} \psi$ configuration. Reflections from the $\{311\}$ lattice plane were measured at $16 \psi$ angles from $-70.5^{\circ}$ to $70.5^{\circ}$ at a $2 \theta$ angle of approximately $78.05^{\circ}$. These were taken at $6 \varphi$ angles $\left(0^{\circ}, 45^{\circ}\right.$ and $90^{\circ}$ and at $180^{\circ}$ to each of these to obtain the negative $\psi$ angles $)$. With these measurements and the plane stress assumption, the in-plane stress tensor could be obtained. At each point, the sample was oscillated over a distance of $2 \mathrm{~mm}$ to improve counting statistics due to the large grain sizes as well as texture in the rolled plate samples. The measurement depth was estimated to be $26 \mu \mathrm{m}$ using the AbsorbDX software.

Incremental Hole Drilling. IHD was performed using the SINT Restan MTS3000 Automatic Hole Drilling Machine for both sample thicknesses. Vishay type A strain gauge rosettes with a rosette diameter of $5.13 \mathrm{~mm}$ and nominal hole diameter of $1.8 \mathrm{~mm}$ were used. The holes were drilled at the centre of the LSP patches and the rosettes were aligned such that gauge 1 was aligned with the $\mathrm{x}$-direction and gauge 3 with the $\mathrm{y}$-direction. Each hole was drilled to a depth of $1.2 \mathrm{~mm}$ in steps of $20 \mu \mathrm{m}$. The residual stresses were calculated according to the method outlined in the ASTM E837-13 standard [7] with calibration coefficients adapted to the particular strain gauge used [8]. It should be noted that the $1.6 \mathrm{~mm}$ thick samples fell outside of this standard for these particular strain gauge rosettes, which were used despite this study due to availability and cost effectiveness. The residual stresses were calculated at depths from $25-975 \mu \mathrm{m}$ with steps of $50 \mu \mathrm{m}$. Since this technique measures macro strains, the appropriate bulk elastic constants were used for the stress calculations. At least two measurements were performed for each case to ensure repeatability.

Neutron Diffraction. Monochromatic angle-dispersive ND was performed at the MPISI diffractometer at Necsa, South Africa. The neutron beam had a wavelength of $1.67 \AA$ and both the incident and secondary slits set to $0.3 \mathrm{~mm} \times 10 \mathrm{~mm}$ which created a matchstick shaped gauge volume. This shape was used to facilitate high depth resolution to capture the high stress gradient near the surface of the samples with as small a gauge volume as possible in the depth direction. The $\{311\}$ lattice plane was measured at a diffraction angle of approximately $85.5^{\circ}$. Strains were measured in the $\mathrm{x}-, \mathrm{y}$ - and z-directions by aligning the samples so that the direction of strain measurement bisected the incident and diffracted beams and with the long dimension parallel to the vertical surface. The gauge volume projected $0.44 \mathrm{~mm}$ into the depth with the sample in this orientation. Measurements were taken in the $6 \mathrm{~mm}$ thick sample at depths from $0.1-0.3 \mathrm{~mm}$ with steps of $0.1 \mathrm{~mm}, 0.5-1.5 \mathrm{~mm}$ with steps of $0.2 \mathrm{~mm}$ and from $2-5.5 \mathrm{~mm}$ with $0.5 \mathrm{~mm}$ steps. In the $1.6 \mathrm{~mm}$ sample, measurements were taken at depths of $0.1-0.4 \mathrm{~mm}$ with steps of $0.1 \mathrm{~mm}$, from $0.6-1.2 \mathrm{~mm}$ with steps of $0.2 \mathrm{~mm}$ and from $1.3-1.5 \mathrm{~mm}$ with steps of $0.1 \mathrm{~mm}$. At each depth, the samples were oscillated parallel to the surface by performing measurements at 5 locations, $0.5 \mathrm{~mm}$ apart. Data acquisition was done against statistical counting instead of the traditional time counting to a set maximum strain uncertainty of $50 \mu \varepsilon$. Entry curves were employed to ensure coincidence between the gauge volume and the sample surface [9]. With measurements at positions close to the sample surfaces, with partially submerged gauge volumes, the $180^{\circ}$ flip approach was performed with the two results averaged to mitigate surface aberration contributions.

The reference lattice spacing and the planar stresses were calculated at each depth according to the plane-stress assumption with the equations outlined in [10]. The elastic constants listed previously were used for the stress calculations.

Synchrotron X-Ray Diffraction. SXRD Measurements were performed at the 6-BM-A beamline at the Advanced Photon Source (APS) at the Argonne National Laboratory, USA. Energy Dispersive Diffraction (EDD) measurements were made with a hardened polychromatic beam, with a maximum energy of $285 \mathrm{keV}$, from an APS bending magnet. Two germanium 
detectors were used, one detector vertically offset to a diffraction angle of $5.0^{\circ}$ and the other horizontally offset to a diffraction angle of $4.8^{\circ}$. This allowed simultaneous measurement of the in-plane and horizontal strain components at each depth. Due to the small diffraction angle of the detectors, the direction of strain measurement is nearly perpendicular to the primary beam. Both the beam size and the detector slits were set to $0.1 \mathrm{~mm} \times 0.1 \mathrm{~mm}$. With this geometry the gauge volume projects to a horizontal length of about $2.6 \mathrm{~mm}$. The sample was aligned in the gauge volume such that the vertical detector measured the in-plane strain component, the horizontal measured the normal strain component and the projected length was parallel to the surface. For the $6 \mathrm{~mm}$ thick sample, measurements were taken at depths from $0.05-2.55 \mathrm{~mm}$ with a depth step of $0.1 \mathrm{~mm}$ and from $3.05-5.55 \mathrm{~mm}$ with a depth step of $0.5 \mathrm{~mm}$. For the $1.6 \mathrm{~mm}$ thick sample, measurements were taken at depths from $0.05-1.55 \mathrm{~mm}$ with a depth step of $0.1 \mathrm{~mm}$. At each depth, the samples were oscillated parallel to the surface by performing measurements at 5 locations, $0.1 \mathrm{~mm}$ apart. The strains were calculated by averaging the data from each location. A counting time of $40 \mathrm{~s}$ was used for each measurement.

To calculate strain from the EDD data, the average lattice parameter can be determined by utilising all the reflections available in the diffraction pattern. However, due to noise in the system, only the peaks of the $\{200\}$ and $\{311\}$ planes could be numerically fitted at every depth. Since the $\{200\}$ plane is prone to inter-granular stresses, only the $\{311\}$ peak positions were used to determine the lattice spacings and, hence, the strain. The reference spacing and the residual stresses at each depth were calculated in the same manner as for neutron diffraction.

\section{Results and Discussion}

Fig. 2 shows the residual stress results obtained using the various methods described for the $6 \mathrm{~mm}$ thick sample in the $\mathrm{x}$-direction, both with and without the LSP treatment. The stresses were also measured in the y-direction (omitted from Fig. 2 due to space restrictions) and the two components of stress had similar trends: a large compressive stress near the surface and tensile stress in the interior for the peened sample and a low stress- profile in the untreated sample. The magnitudes of the stresses were anisotropic being approximately $20 \%$ more compressive in the $\mathrm{x}$-direction (laser step) than in the y-direction (laser scan). This compares well to results in literature $[1,5]$.

The ND and Laboratory XRD investigations on the unpeened sample showed nearly zero residual stress. In the peened sample the ND and SXRD results show corresponding residual stress profiles through the entire depth of the sample. This suggests that the approach used to determine the residual stresses was sound, especially considering the different gauge volumes used.

The stresses vary rapidly in the near surface region. The LXRD (depth of $26 \mu \mathrm{m}$ ) and IHD (depth of $25 \mu \mathrm{m}$ ) results showed compressive residual stresses of approximately -200 $\mathrm{MPa}$, whilst SXRD (depth of $50 \mu \mathrm{m}$ ), IHD (depth of $150 \mu \mathrm{m}$ ) and ND (depth of $160 \mu \mathrm{m}$ ) all showed stresses in excess of $-350 \mathrm{MPa}$. The stress relaxation close to the surface may be attributed to the laser-material interaction at the surface causing reverse yielding in these regions.

Although IHD is typically not accurate near to surfaces, it does correspond well with the Laboratory XRD results.

These results reveal the surface residual stress, the maximum value of the compressive residual stress, as well as the depth to which the residual stresses are compressive. These could not have been attained with only one method which strongly supports the argument that a residual stress profile is best obtained using a variety of methods.

The fairly large error bars on the Laboratory XRD results are due to texture, as observed from the Debye-Scherer cones on the area detector. At certain $\psi$ angles, the X-Ray counts were 
insufficient for peak fits. Further evidence of texture in the samples came from the ND and SXRD experiments.

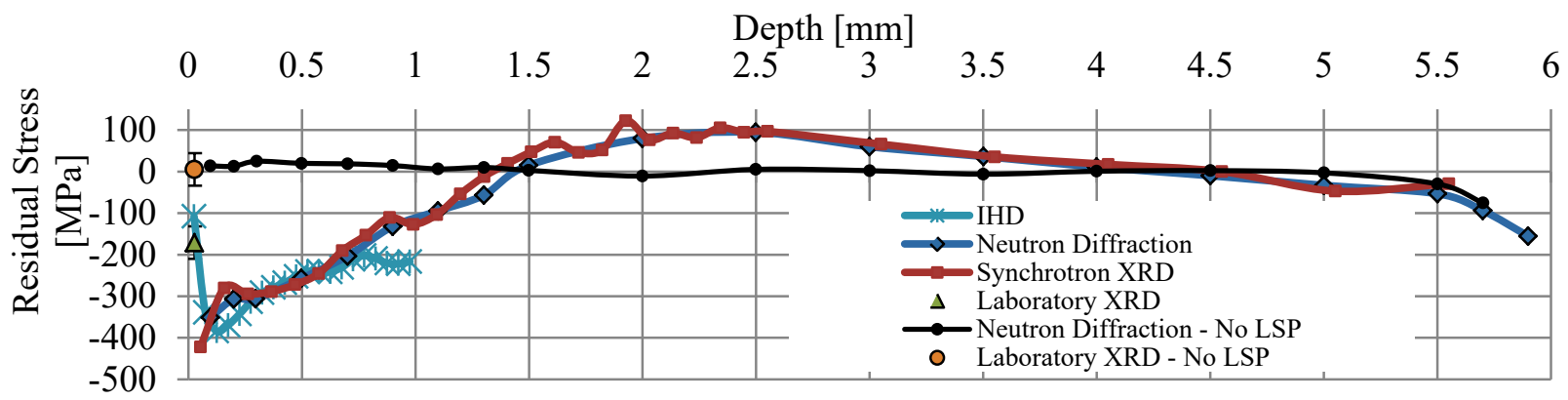

Fig. 2: Depth-resolved residuals stress results in the $x$-direction obtained from multiple methods on the $6 \mathrm{~mm}$ thick sample with and without LSP treatment.

Fig. 3 shows the residual stress results in the x-direction obtained for the $1.6 \mathrm{~mm}$ thick sample with LSP. Again there is good correlation between the ND and SXRD results. The residual stresses are substantially less compressive than in the $6 \mathrm{~mm}$ thick sample. This is because there is less elastic constraint in this sample.

An offset is observed between the IHD result and the SXRD and ND below $400 \mu \mathrm{m}$. This can be attributed to the $1.6 \mathrm{~mm}$ being too thin for application of the ASTM E837 standard. It is proposed that thickness-specific calibration coefficients should be developed for samples falling outside of the standard.

Surface relaxation could not be detected with the ND and SXRD results.

It is important to note than the residual stresses are compressive through the whole depth of the sample. This means that elsewhere in the sample there will be balancing tensile stress and this needs to be considered when designing an LSP treatment - care must be taken to avoid placing a tensile residual stress in a fatigue critical location.

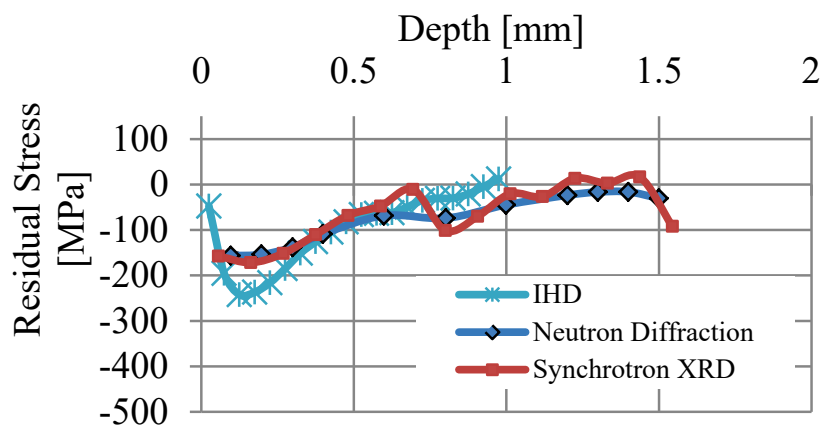

Fig. 3: Depth-resolved residuals stress results in the $x$-direction obtained from multiple methods on the $1.6 \mathrm{~mm}$ thick sample with LSP treatment.

\section{Conclusions}

Residual stresses in $6 \mathrm{~mm}$ and $1.6 \mathrm{~mm}$ thick aluminium alloy 7075 samples that had been treated with LSP have been measured using various complementary techniques. The results show high magnitude compressive residual stresses $(-200--400 \mathrm{MPa})$ with steep gradients. Stress relaxation was shown to occur near the surface. Larger compressive residual stresses were established in the $6 \mathrm{~mm}$ thick sample because there was more elastic constraint provided by the underlying material volume. Additionally, the residual stresses were more compressive in the laser step direction than in the laser scan direction.

Residual stress results from the various methods employed in this study show complementary trends. Since each method has its strengths and limitations, this work showed the necessity of 
using multiple complementary techniques to fully describe the depth-resolved residual stress profile associated with LSP treatment.

\section{Acknowledgements}

The authors would like to acknowledge the DST-NRF Centre of Excellence in Strong Materials (CoE-SM) for their generous financial support. They would also like to acknowledge the vital support of the South African Centre for Scientific and Industrial Research National Laser Centre's (CSIR NLC) Rental Pool Program (RPP), funded by the Department of Science and Technology (DST).

In addition, this work is based on the research supported in part by the National Research Foundation (NRF) of South Africa Incentive Funding for Rated Researchers (IFRR) for Prof. Claudia Polese and Equipment-Related Travel and Training Grant (ERTTG) (Grant Numbers: 109200 and 115195).

Opinions, findings and conclusions or recommendations expressed in this work are those of the authors and are not necessarily to be attributed to the CoE-SM or to the NRF.

\section{References}

[1] K. Ding and L. Ye, Laser Shock Peening Performance and Process Simulation, Woodhead Publishing Limited, Cornwall, 2006. https://doi.org/10.1201/9781439823620

[2] T. Adachi et al., Effect of laser peening on fatigue properties for aircraft structure parts, in: International Conference on Shot Peening, 2008.

[3] Y. Sano, M. Obata, T. Kubo, N. Mukai, M. Yoda, K. Masaki and Y. Ochi, Retardation of crack initiation and growth in austenitic stainless steels, Mater. Sci. Eng. A, 417 (2006) 334-340. https://doi.org/10.1016/j.msea.2005.11.017

[4] Q. Yu, Z. Dong, R. Miao, X. Deng and L. Chen, Bending deformation of laser peened aluminium alloy with uniform rectangular spots, Mater. Sci. Technol. 32 (2016) 9-14. https://doi.org/10.1179/1743284715Y.0000000057

[5] C.A. Rodopoulos, J.S. Romere, S.A. Curtis, E.R. de los Rios and P. Peyre, Effect of controlled shot peening and laser shock peening on the fatigue performance of 2024-T351 aluminum alloy, J. Mater. Eng. Perform. 12(4) (2003) 414-419. https://doi.org/10.1361/105994903770342944

[6] N.S. Rossini, M. Dassisti, K.Y. Benyounis and A.G. Olabi, Methods of measuring residual stresses in components, Mater. Des., $32 \quad$ (2012) 572-588. https://doi.org/10.1016/j.matdes.2011.08.022

[7] ASTM, ASTM E837 - 13a Standard test method for determining residual stresses by the hole-drilling strain-gage method, ASTM International, West Conshohocken, 2013.

[8] E. Valentini, A. Benincasa and L. Bertelli, Residual stress calculation using the incremental hole-drilling method with eccentric holes: the eval software, in: Associazione Italiana Per L'analisi Delle Sollecitazioni $43^{\circ}$ Convegno Nazionale, Rimini, 2014.

[9] P.C. Brand and H.J. Prask, New methods for the alignment of instrumentation for residualstress measurements by means of neutron diffraction, J. Appl. Crystallogr. 27 (1994) 164-176. https://doi.org/10.1107/S0021889893007605

[10] A.M. Venter, D. Marais and V. Luzin, Benchmarking studies of the MPISI Material Science Diffractometer at SAFARI-1, Mat. Res. Proc. 2 (2016) 413-418. 